\title{
Numerical Simulation on Conical Shaped Charge with Copper Liner in Several Typical Shapes
}

\author{
Z Liu*, J Zhai, S Su \\ State Key Laboratory of Explosion Science and Technology, \\ Beijing Institute of Technology, China
}

\begin{abstract}
Jet formation and target penetration capabilities from conical shaped charges with liner geometry of five types: (1) cone, (2) round-tipped cone, (3) hemisphere, (4) ellipsoid, and (5) trumpet, have been simulated via LSDYNA Multi-Materials Arbitrary Lagrangian Eulerian (MMALE) technique. The objective is to observe the influences of liner types on the penetration features in target block while the amount of explosive and liner material are identical in all charge cases. Simulation results show that the hemispherical liner brings out the lowest penetration, while the ellipsoid and trumpet liners are of middle performance, but, the cone and round-tipped cone liners exhibit best penetration. Shaped charges with ellipsoid liner and trumpet liner present no remarkable discrepancy on penetration depth, however, the entrance holes are dramatically different in geometrical shape. Such penetration features are anticipated to be useful in technical design of shaped charges for some specific applications.
\end{abstract}

\section{INTRODUCTION}

Jet from shaped charge is of important role in penetration, cutting, perforation, and other applications [1]. Device of shaped charge is composed of two main parts: explosive filling and metallic liner. Traditionally, the conical shaped charge with conical copper liner has been utilized broadly. In the later development there appeared the effort to test other geometrical form liners. Fedorov et al. [2] once proposed a liner with form of hemisphere-cylinder combination used for a shaped charge setup. Cao et al. [3] made numerical simulation on a shaped charge with a liner of hemisphere form and gained the result of relatively low jet tip velocity. In this paper, instead of experimental investigations, numerical exploration is undergone to systematically study the performance of shaped charges with five kinds of liner forms in geometry: (1) cone, (2) round-tipped cone, (3) hemisphere, (4) ellipsoid, and (5) trumpet. The so-called Multi-Materials Arbitrary Lagrangian Eulerian (MMALE) numerical technique in LS-DYNA software [4] is employed for accomplishing all numerical simulations. Owing that all above shaped charges are of the structural feature of two-dimensional axial symmetry, the 2D geometrical arrangement has been considered in the simulation. The effort is devoted to compare the penetration depths into the target block, and the sizes of the craters caused after jet entrance. These features may provide useful support for the design of shaped charge for some special applications. 


\section{SIMULATION PROCEDURES}

2.1. Computational model.

Conical shaped charge as depicted in Figure 1 is based on the prototype from Reference [5]. It basically consists of a liner, an explosive filling, and an outer case. Liner material is copper with thickness of $0.2 \mathrm{~cm}$. The outer case is an aluminium body being $0.2 \mathrm{~cm}$ in thickness as well. TNT high explosive is used for charge filling. Other dimensions relevant to the device are given in Table 1. To witness the penetration capability, a steel target is set away at some stand-off distance off the bottom of the shaped charge. Steel target is of a geometry of solid cylinder with $15.24 \mathrm{~cm}$ in diameter by $62.87 \mathrm{~cm}$ long. Stand-off distance is set to be $20 \mathrm{~cm}$. All computational models are pre-processed by Hypermesh tool for computation mesh configuration. Totally five liner forms: cone, round-tipped cone, hemisphere, ellipsoid and trumpet, are respectively considered in each shaped charge model.

Table 1: Basic geometrical parameters for shaped charge model.

\begin{tabular}{llll}
\hline Liner thickness & $\begin{array}{l}\text { Height of expl. filling } \\
\mathbf{h}(\mathbf{c m})\end{array}$ & $\begin{array}{l}\text { Diameter } \\
\mathbf{d}(\mathbf{c m})\end{array}$ & $\begin{array}{c}\text { Apex angle } \\
\boldsymbol{\alpha}\left({ }^{\circ}\right)\end{array}$ \\
0.20 & 5.29 & 10.00 & 60 \\
\hline
\end{tabular}

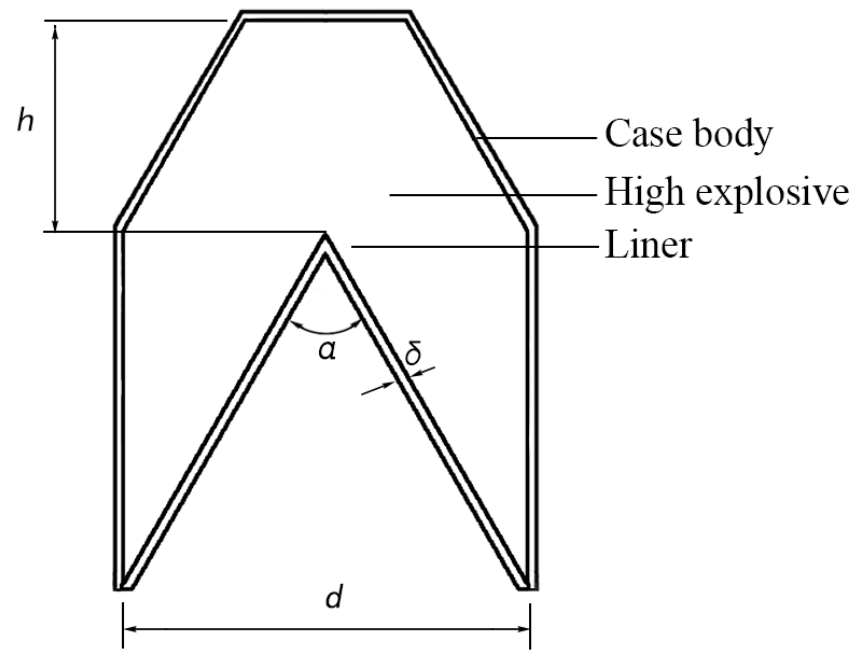

Figure 1: Basic configuration of conical shaped charge considered for numerical simulation.

The simulation goes to include the processes of jet formation from shaped charge, and the ensuing jet penetration into steel block. Figure $2 \mathrm{a}$ aligns the arrangement with the presence of a charge model with cone form liner. Figures $2 b$ through $2 \mathrm{e}$ give the remaining liner forms together with charge shapes in a manner of symmetrical cross section. In the practical calculation, only half part of Figure 2a (upper or lower along the symmetrical line) need to 
be considered due to the feature of the symmetry. In all cases, in order to hold the weight of the explosive filling being identical, the dimensions of the outer aluminium body have been slightly adjusted in accordance with different liner form.

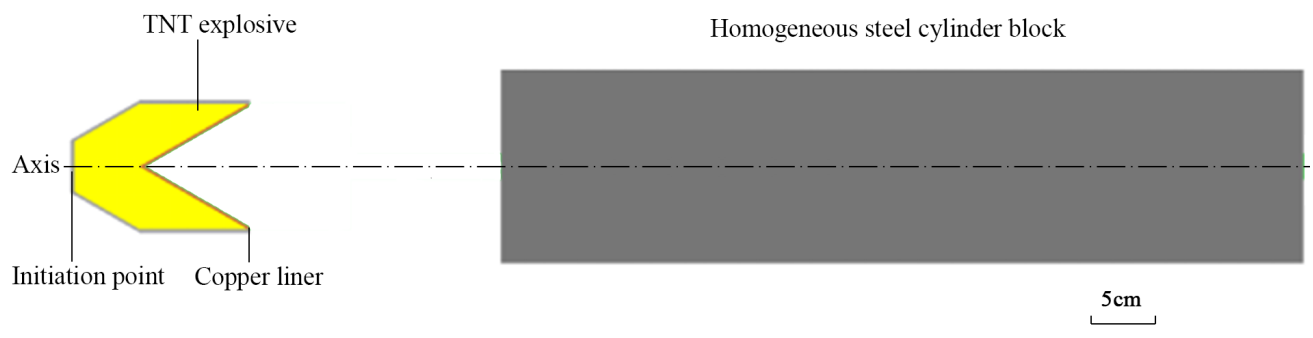

(a) Cone liner

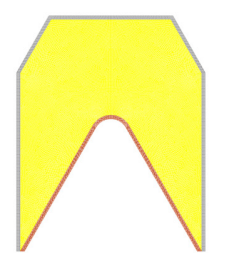

(b) Round-tipped cone liner

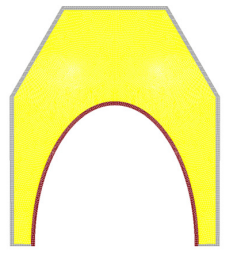

(d) Ellipsoid liner

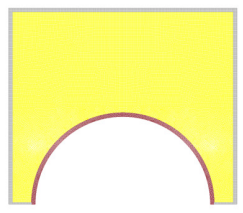

(c) Hemisphere liner

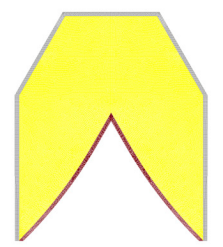

(e) Trumpet liner

Figure 2: Alignment of computational model for shaped charge setup and witness steel block, with each liner form being illustrated.

\subsection{Computational method.}

Multiple Materials Arbitrary Lagrangian Eulerian (MMALE) solver in LS-DYNA software is used for the whole computations. Both the processes of charge detonation and jet formation are simulated by MMALE completely, however, simulating jet penetration into steel block, 
the fluid-structure coupling method is employed while jet being of fluid and target being of Lagrangian structure. Once jet impinges the target, the fluid structure coupling is activated automatically for the calculation of the later penetration. Mesh size of $0.05 \mathrm{~cm}$ is set by a preliminary calculation with good stable result. This mesh size has been adopted for all models. Quadrilateral meshes are utilized in all calculations in order to gain the better computational stability

Point initiation method is employed for triggering the detonation of the explosive in charge with the starting point at the top of the charge setup. The ensuing detonation propagation is modelled by a programmed burn, in which the product of time and detonation speed determines a position in detonation or not.

\subsection{Equations of state and material models.}

The simulations involve multi-materials such as fluid and solid, hence, equations of state and strength model equations for respective materials are in necessity. For the high explosive, Jones-Wilkins-Lee (JWL) form equation of state is employed. The property parameters for TNT can be found from the explosive handbook as of Reference [6]. The liners in all charges are of the same material of copper, with elastic-plastic deformation feature in dynamic loading. Steinberg strength model [7] is such a choice for description of elastic-plastic deformation involving factors of strain, strain rate, and temperature. For high pressure state, Mie-Gruneisen equation of state is used for solid material. The relevant parameters for copper and steel can be obtained from Reference [8]. Finally, the target is treated as dual-linear elastic-plastic material containing the criterion for a failure judgment, in which once the equivalent strain in target becomes greater than the criterion strain for material failure, it is believed that the material at that point is broken. Correspondingly, when an element is at such state, it will be deleted in the later phase of computation. The model parameters used for steel target are from Reference [5].

\section{RESULTS AND DISCUSSION}

At the beginning, the shaped charge with cone liner form was computed because of its baseline role. The calculated results are presented in Figure 3 containing several typical configurations such as jet formation, jet impinging upon target, and initial, middle, final penetrations in target block. From the figure, it is illustrated that at $15 \mu \mathrm{s}$ instant after TNT is initiated and detonated, liner begins to collapse by the propel of high-pressure detonation products, giving an initial jet shape. When time is at $62 \mu \mathrm{s}$, the jet almost touches the target block just as Figure 3(b) shown. Jet and slug are visibly distinguishable at this moment with jet moving ahead and slug lagging behind. Afterwards, jet continues to stretch even it strikes into target. Figure 3(c) shows the initial penetration of jet into target accompanying a big entrance hole. In the later phases of penetration, jet drops down in velocity, and the mass of jet is consumed by dispersion on the surface of penetrating hole. Finally, jet loses the penetration ability because of slow velocity and less jet mass. It is hindered at the bottom of the hole as Figure 3(e) shows. At the same time, there is a departure of slug and jet during penetration. It can be seen from Figure 3(d) clearly.

Similarly, the charges with the other four liners have also been simulated for observing jet formation and penetration. The detailed computational results are not illustrated, and only typical features are compared for those models. Firstly, the jet tip velocities before striking the target are collected and presented in Figure 4 for comparison. In all five cases, shaped 


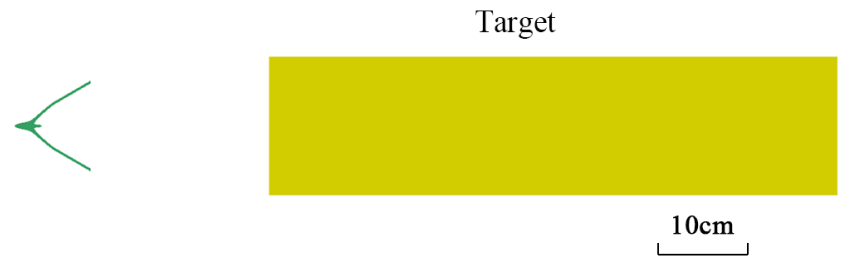

(a) $15 \mu \mathrm{s}$

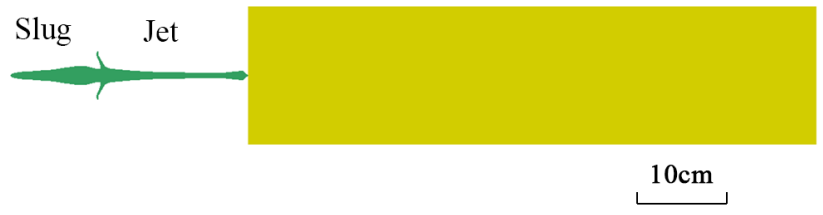

(b) $62 \mu \mathrm{s}$

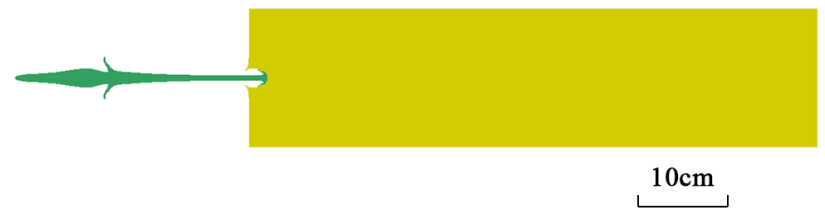

(c) $69 \mu \mathrm{s}$

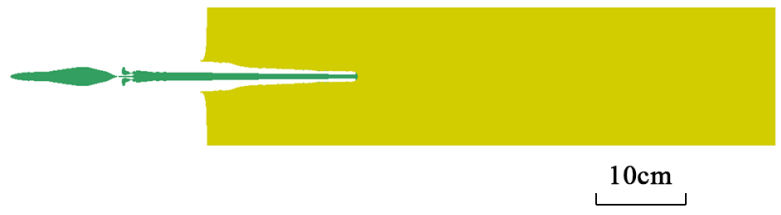

(d) $140 \mu \mathrm{s}$

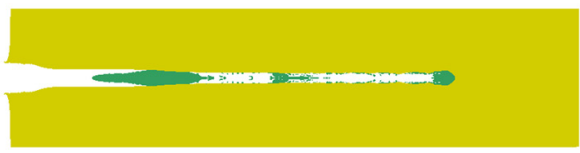

$10 \mathrm{~cm}$

(e) $700 \mu \mathrm{s}$

Figure 3: Simulated results of jet formation and penetration into target for shaped charge with cone liner form.

charge with round-tipped cone liner form provides the maximum jet velocity, then, following by the case of cone form liner. Trumpet form liner also provides a higher jet velocity relatively, 
but ellipsoid form liner brings out a rather slower jet velocity, with hemisphere form liner produces lowest, only a little higher than half value of jet velocity by the round-tipped cone form liner. In addition, on the fastness for jet impinging into the target, the charge with trumpet form liner underwent a shortest time period, following by the cases of cone and round-tipped cone liners. However, the ellipsoid and hemisphere liners exhibit much slower arrival times for jet to strike the target. The reason for such difference is that charges with ellipsoid and hemisphere liners need a longer time for liner collapsing to form jet due to the larger separations in liner geometry.

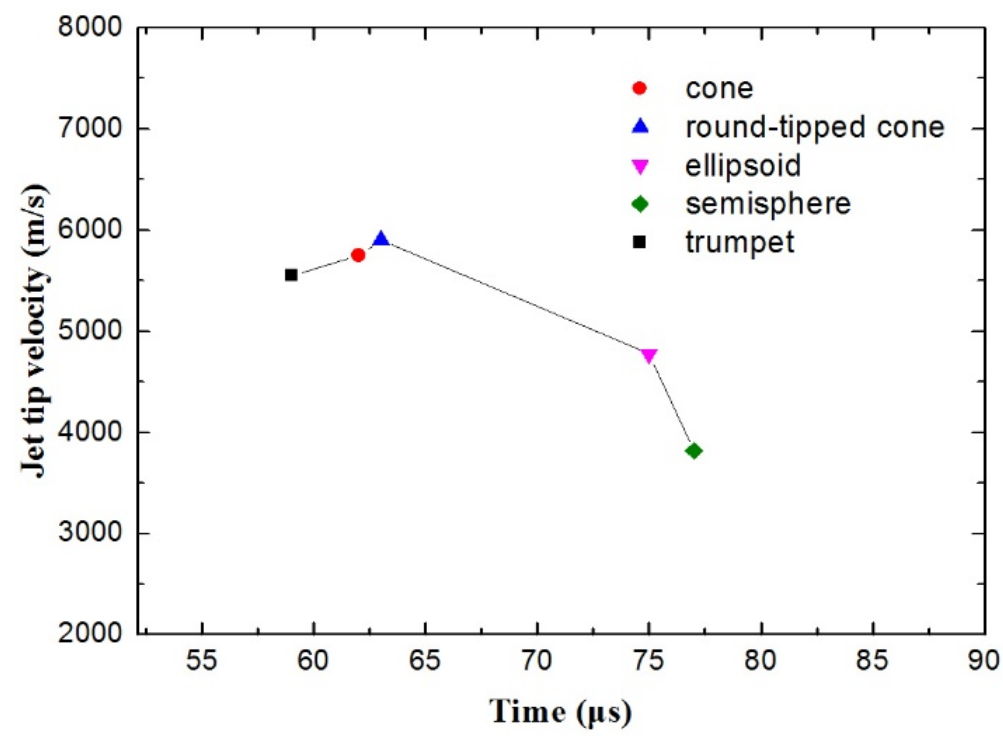

Figure 4: Jet tip velocities by shaped charges with various geometrical liners just before striking the target.

Regarding the penetration process, jet tip velocity cannot solely determine the depth of final penetration. It is the kinematic energy of the whole jet that determines the penetration depth. Kinematic energy of jet is related to mass distribution of jet as well as its corresponding velocity. Figure 5 shows the computed jet configurations for five charges with each liner form in respective shaped charge before striking the target. The colour variations, from blue to red colour band in the figure, denote the velocity magnitudes from $0.4 \mathrm{~km} / \mathrm{s}$ to $6.3 \mathrm{~km} / \mathrm{s}$ correspondingly. The colour expression clearly exhibits the velocity gradients in jet and slug during jet motion. The existence of velocity gradient would cause jet stretching, forcing jet to become slimmer. The jet tip velocities in all five shaped charge models are also visibly understood by colour difference in Figure 5. Charges with cone, round-tipped cone and trumpet liners produce a relatively higher tip velocity, and cases with hemisphere and ellipsoid liners are, however, of lower jet tip velocity. On the other hand, the charges with hemisphere and ellipsoid liners, can generate jet with extremely large diameter, implying more mass distribution in jet portion. 


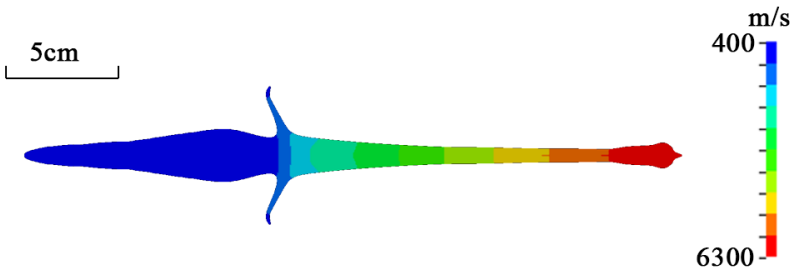

(a) Cone liner case

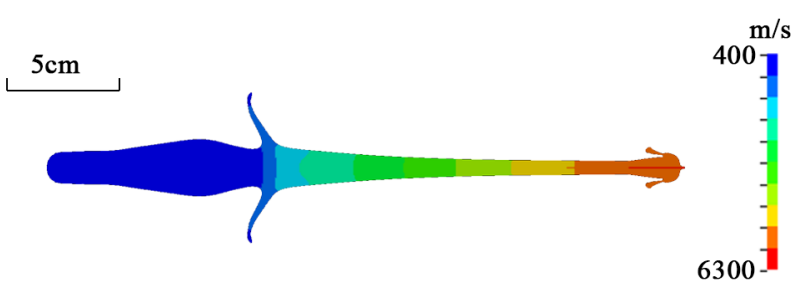

(b) Round-tipped cone liner case

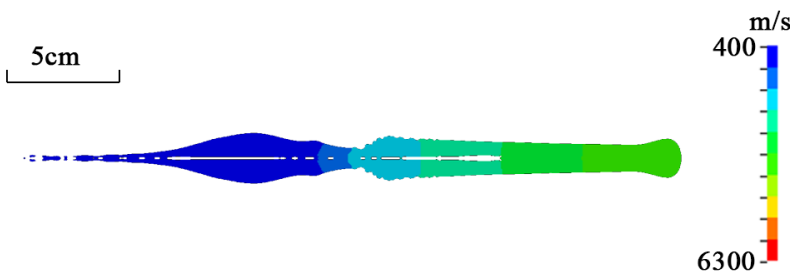

(c) Hemisphere liner case

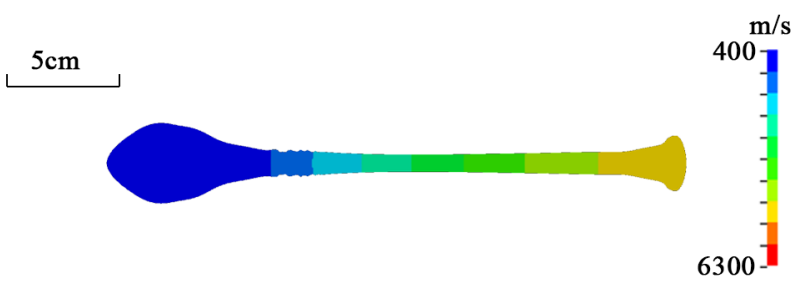

(d) Ellipsoid liner case

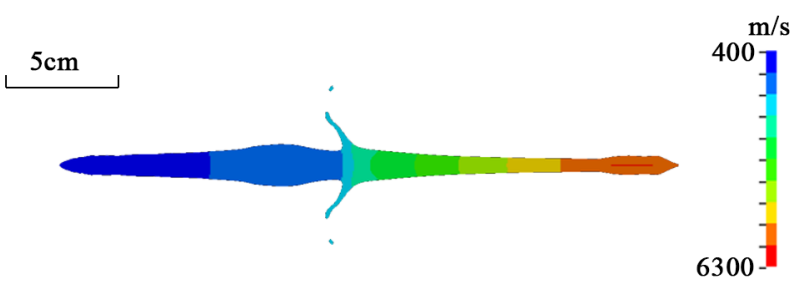

(e) Trumpet liner case

Figure 5: Distributions of velocity and mass of jet before striking the target from five charge models. 


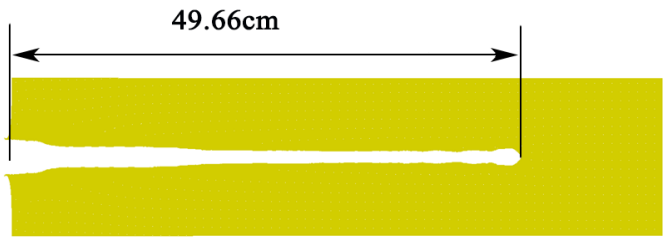

(a) Cone liner case

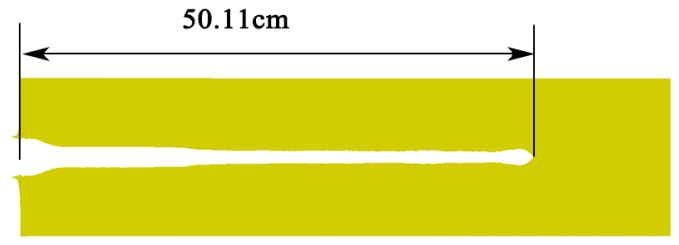

(b) Round-tipped cone liner case

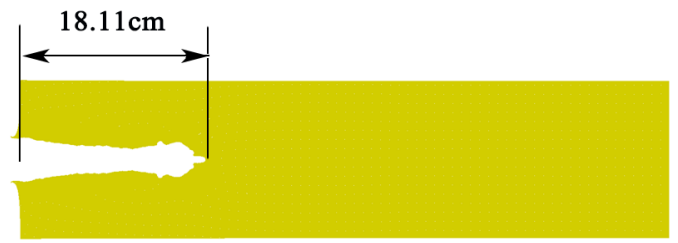

(c) Hemisphere liner case

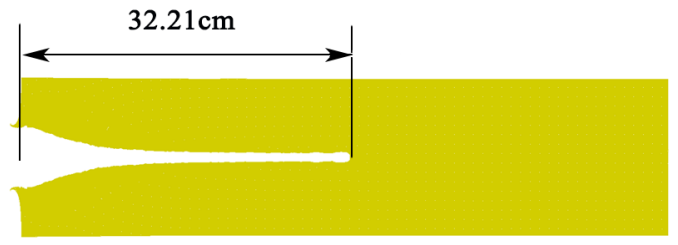

(d) Ellipsoid liner case

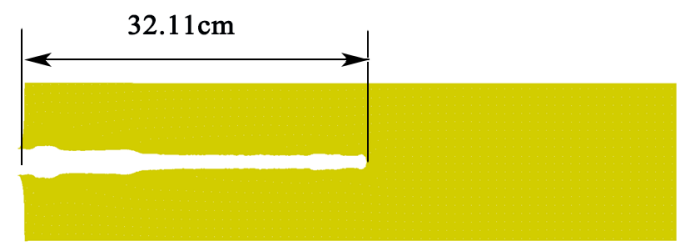

(e) Trumpet liner case

Figure 6: Final penetration depths by shaped charges with liners in different geometries. 
On penetration depth, the data are tabulated in Table 2 for all five shaped charge models. Charge with hemisphere liner generates the lowest penetration depth of $18.11 \mathrm{~cm}$, however, charges with cone and round-tipped cone liners can achieve the depth of around $50 \mathrm{~cm}$. The moderate and similar penetration depths by charges with ellipsoid and trumpet liners are $32.21 \mathrm{~cm}$ and $32.11 \mathrm{~cm}$, respectively.

Table 2: Calculated results of penetration depth by charges with different liner forms.

\begin{tabular}{llllll}
\hline Liner shape & Cone & $\begin{array}{l}\text { Round-tipped } \\
\text { cone }\end{array}$ & Hemisphere & Ellipsoid & Trumpet \\
\hline Penetration depth $(\mathrm{cm})$ & 49.66 & 50.11 & 18.11 & 32.21 & 32.11 \\
\hline
\end{tabular}

Figure 6 shows the distribution of penetration holes by jet from five liner shaped charges, as well as hole depth in magnitude. Charges with cone and round-tipped cone liners produce holes with similar geometrical appearance and close penetration depth. Charges with ellipsoid liner and trumpet liner may produce very similar penetration depth, but the craters at jet entrance are remarkably different. It implies that if large crater at jet entrance is desirable in application, charge with ellipsoid liner is a recommendable device. Moreover, charge with hemisphere liner produce the shortest penetration depth, however, the total penetration hole is of large diameter, meaning that if uniformly large hole is required, this device is an ideal choice.

\section{CONCLUSIONS}

Jet formation and characteristics of target penetration from conical shaped charges with varied liner forms have numerically been simulated via LS-DYNA dynamic software. The following conclusions can be achieved:

1. Under the same mass of explosive, the traditional charge design with cone form liner is able to produce the ideal penetration depth for targets. Other varied designs do not provide better penetration capability.

2. Charge with ellipsoid form liner may cause a larger crater at jet entrance, and charge with hemisphere form liner is able to produce a uniformly large hole. These features provide useful information in designing shaped charge for special applications.

\section{REFERENCES}

[1] Walters, W. and Zukas, J., Fundamentals of Shaped Charges, 1989, John Wiley \& Sons, New York.

[2] Fedorov, S.V., Bayanova, Ya.M. and Ladov, S.V., Numerical analysis of the effect of the geometric parameters of a combined shaped-charge liner on the mass and velocity of explosively formed compact elements, Combustion, Explosion, and Shock Waves, 2015, 50(1), 130-142.

[3] Cao, L., et al., Numerical Simulation of Effect of Liner Structure on Performance of Shaped Charge Jet, MINING R\&D, 2009, 29(6), 98-100.

[4] LS-DYNA Manual R.9448, 2018, Livermore Software Technology Co.

[5] Shi, D., Li, Y. and Zhang, S., Explicit Dynamic Analysis Based on ANSYS/LS-DYNA, 2005, Tsinghua University Press, Beijing. 
[6] Dobratz, B.M. and Crawford, P.C., LLNL Explosives Handbook, 1985, Lawrence Livermore National Laboratory Report, Livermore, CA.

[7] Steinberg, D.J., Cochran, S.G. and Guinan, M.W., A Constitutive Model for Metals Applicable at High Strain Rate, J. App. Phys., 1980, 51(3), 1498-1504.

[8] Katayama, M., Kibe, S. and Yamamoto, T., Numerical and Experimental Study on the Shaped Charge for Space Debris Assessment, Acta Astronautica, 2001, 48 (5-12), 363372. 